\title{
$K$-shell binding energy of Be and its fluorescence yield
}

\author{
Cleanthes A. Nicolaides and Yannis Komninos \\ Theoretical and Physical Chemistry Institute, National Hellenic Research Foundation, \\ Athens 501/1, Greece \\ Donald R. Beck \\ Physics Department, Michigan Technological University, Houghton, Michigan 49931
}

(Received 24 January 1983)

\begin{abstract}
The smallness of $\mathrm{Be}$ and its valence structure are attractive characteristics for the rigorous application and testing of the flexibility and utility of many-body theories. Recent$1 \mathrm{y}$, a number of results of many-electron calculations have been published on the $1 \mathrm{~s}$ binding energy $(B E)$ and the Auger width $(\Gamma)$ of the hole state. They show considerable dispersion. We have studied these properties and the fluorescence yield $\left(\omega_{K}\right)$ of $\mathrm{Be}^{+} 1 s 2 s^{2} S$ by applying a general theory of autoionizing states which is based on projected function spaces and justifies the variational calculation of localized relativistic or nonrelativistic correlation and the subsequent inclusion of the continuum. Two computational methods have been employed: The first method (A) aims at the accurate calculation of the total energy of initial and final states. In the case of $\mathrm{Be}$, its ground-state energy is known accurately. The excited-state energy is computed in this work variationally and includes the effect of the continuum. The resulting correlated square-integrable wave function $\Psi_{0}$ of the hole state is then employed for the calculation of the Auger width and the radiative transition probabilities to the lower discrete states $1 s^{2} 2 p$ and $1 s^{2} 3 p^{2} P^{o}$. We find that the angular correlation increases the decay rates whereas inclusion of radial and spin-polarization correlation eventually decreases them by a factor of 2 from the Hartree-Fock (HF) value. Nonorthonormality (NON) is taken into account explicitly; we show that radiative decay does not arise only from correlation effects. The second method (B) is based on the consistent analysis of electronic structure and aims at the calculation of only those one- and two-subshell correlations which contribute to the BE the most. The validity and generality of the second methodwhich has been applied before to a number of atoms in the periodic table-is demonstrated once again. Our results are, with method $\mathrm{A}, \mathrm{a} B \mathrm{BE}$ of $123.732 \mathrm{eV}, \Gamma=0.023 \mathrm{eV}$, and $\omega_{K}=1.2 \times 10^{-4}$ and, with method B, a BE of $123.82 \mathrm{eV}$. The BE's include an empirical relativistic correction of $0.027 \mathrm{eV}$. An Auger-spectroscopy measurement has yielded a BE of $123.6 \pm 0.1 \mathrm{eV}$. Our value for $\omega_{K}$ is closer to the available experimental values from the solid state $\left(\approx 3.3 \times 10^{-4}\right)$ than a previous many-electron calculation, by a factor of 5 .
\end{abstract}

\section{INTRODUCTION}

During the past 15 years or so, photoemission spectroscopy ${ }^{1-3}$ has made significant advances in probing the electronic structure of matter and its response to uv and $\mathrm{x}$-ray radiation. The measurement of one-electron binding energies (BE) and of Auger energies and widths is an integral part of this field. The calculation of these quantities has been dealt with through a variety of many-body approaches (e.g., Refs. 4-11 and references therein).

The purpose of this paper is to present new and accurate results on the $1 s \mathrm{BE}$ Auger width and fluorescence yield of Be and discuss certain aspects of the theory of such calculations. Our results are compared with those of other theoretical methods $^{5-10}$ and experiment. ${ }^{12-14}$

The standard theoretical definition of $\mathrm{BE}$ is the following: total energy of final state minus total energy of initial state. Although this constitutes a rigorous definition, it may not always correspond exactly to the observed $\mathrm{BE}$, due to possible details of the dynamics of excitation and measurement which, in principle, could show up as a function of photon energy. ${ }^{11}$

Taken as differences of total energies with respect to a particular relativistic Hamiltonian (e.g., Dirac plus Breit or Møller operators), the $\mathrm{BE}$ can be analyzed in terms of Dirac-Fock (DF) or HF, electron correlation, relativistic correlation, and radiative contributions. ${ }^{4,11}$ The accurate treatment of the last two terms for a general many-electron system 
has not yet been accomplished. The sections which follow essentially deal with the first two terms. Although the calculations on Be, reported in Sec. III, are nonrelativstic, the remarks of Sec. II refer to relativistic systems in general, where questions of projected spaces and variational calculations are thought of in terms of electron as well as positron occupied or virtual spinors.

\section{THEORY OF INNER-HOLE AUTOIONIZING STATES}

The final state in a BE measurement often lies in one or more continuua of the same symmetry (relativistically, both states lie in the positron continuua too), which then requires that a modified approach (e.g., use of projection operators) be used to accurately deduce the properties associated with such states.

Given the convenience of choosing electronic square-integrable wave functions to describe such states approximately, one must first invoke the formal separation of discrete from continuum vector spaces. Their subsequent interaction yields mixed states which may cause phenomena of interference.

The conceptual similarity of these situations suggests that the formal and practical solutions follow similar techniques. Thus the general concepts of projected spaces and square-integrable boundary conditions on many-dimensional differential equations ${ }^{15,16}$ find their straightforward numerical implementation in the numerical solutions of the HF or DF equations for atoms. The specific boundary conditions which are imposed yield bound-electron solutions which exclude electron or positron continuum components.

On the other hand, if one wishes to develop methods for including relativistic or nonrelativistic electron correlation, a variational approach which employs analytic virtual spaces with variable parameters must consider and resolve, conceptually as well as computationally, the problem of variational collapse (see Refs. 15-18 and 5).

The analysis and justification of the variationperturbation treatment of many-electron autoionizing states has been presented in Refs. 15 and 16. Along these lines, a many-electron theory using complex coordinates has also been developed. ${ }^{15,19}$ In this approach, the zeroth-order approximation of the square-integrable $N$-electron function is a Hartree-Fock, multiconfigurational HF (MCHF), or DF vector. Convergence problems in diffuse (mainly negative-ion) resonances are solved by parametrizing the potential in every intermediate selfconsistent field (SCF) iteration by using noninteger nuclear charges (for the attraction integrals) and noninteger electron charges (for the repulsion in- tegrals). The advantage of using true Hartree-Fock functions for describing, in zeroth order, highly excited states, has been demonstrated again recently, in studies of $\mathrm{He}^{-}$Feshbach and shape resonances ${ }^{20-22}$ and of multielectron photoionization processes. ${ }^{23}$

Once the HF (DF) function has been obtained, localized electron correlation can be introduced into the square-integrable $N$-electron function variationally, by keeping the correlation vectors orthogonal to specific zeroth-order HF (or DF electron or positron) solutions of the function space which must be projected out. This procedure yields an upper bound to second order, ${ }^{16}$ a satisfactory fact, given that the orbital approximation is at the HF level.

If the total wave function $\Psi(E)$ is formally expanded in terms of a subshell cluster expansion, it has the form (given the previous remarks, we now refer to nonrelativistic treatments)

$$
\Psi(E)=\Phi_{\mathrm{HF}}+\Phi_{\mathrm{HF}}^{-1} \sigma+\Phi_{\mathrm{HF}}^{-2} \pi+\Phi_{\mathrm{HF}}^{-3} \tau+\cdots,
$$

where $\sigma$ represents the single-orbital excitations, $\pi$ the orbital pair excitations, and $\tau$ the orbital triple excitations. The correlation functions, $\pi, \tau$, etc. contain, in general, closed as well as open channels.

Cluster expansions of the $N$-electron wave function were introduced into atomic and molecular physics by Sinanoglu ${ }^{24}$ in terms of spin orbitals. Along these lines, extremely useful concepts were critically analyzed and applied, such as the independent pair approximation and transferability of pair correlation during a chemical or physical process. $^{24-26}$ Application of this theory allowed the first prediction of inner-electron binding and Auger energies in open-shell neutral and negative-ion atoms. $^{27}$

The subshell analysis of the $N$-electron function is more suited for spectroscopic studies. It does not have a one-to-one correspondence with the spinorbital expansion. For example, single-orbital excitations may contain, due to symmetry, two spinorbital excitations. Thus their energy contributions can be significant-as in the case of $\mathrm{Be} 1 s 2 s^{2} S$ examined here.

Since $\Psi(E)$ belongs to the continuous spectrum, the correlation functions $\sigma, \pi, \tau, \ldots$ contain both closed and open channels. The corresponding correlation can be called localized (loc) and asymptotic (as), respectively. For most hole states, $\sigma$ has only closed channels. Thus we can write

$$
\begin{aligned}
& \sigma=\sigma_{\mathrm{loc}}, \\
& \pi=\pi_{\mathrm{loc}}+\pi_{\mathrm{as}}, \\
& \tau=\tau_{\mathrm{loc}}+\tau_{\mathrm{as}} .
\end{aligned}
$$


For isolated autoionizing states, our theory assumes that localized and asymptotic correlation can be decoupled to a very good approximation and computed with different methods. For localized correlation we employ ordinary variational methods subject to appropriate orthogonality constraints. ${ }^{15,16}$ The asymptotic correlation is included afterwards from a configuration interaction in the continuum approach. $^{28}$

\section{THE $1 s$ BE OF Be}

Because of its configurational simplicity, Be offers a good case for an accurate numerical application of the basic concepts of our approach. At the same time, it has characteristics which are general enough to allow comparison with more complicated systems. Consider the following, for example.

(a) It has strong Fermi-sea correlations in the $L$ shell, $2 s^{2} \leftrightarrow 2 p^{2}$, which affect the $K$-shell excitation and Auger spectrum. The influence of valence multiplet structure and correlations on the spectra of inner electrons is a general characteristic throughout the periodic table and must be included in manybody calculations.

(b) The opening of a hole in the $K$ shell increases the effective charge seen by the $L$-shell electrons. Thus, even though the structure of the neardegeneracy correlations is the same for initial and final states $\left(2 s^{2} \leftrightarrow 2 p^{2}\right)$, their energy contribution is different and, therefore, when computed properly, does not subtract out when taking energy differences. This effective- $Z$ dependence of the Fermi seas persists throughout the periodic table but to different degrees, depending on the shell structure and position of the hole in the system under examination.

(c) The hole-filling localized and asymptotic correlations of the $L$ shell are $2 s^{2} \leftrightarrow 1 s n s, 1 s \in s$. Since these are not of the symmetric-exchange-ofsymmetry (SEOS) type, ${ }^{4}$ i.e., $l^{2} \leftrightarrow(l-1)(l+1)$, they are expected to be small. Our calculations of the energy width and shift bear this out and explain why calculations with only projected square-integrable basis sets yields energies in agreement with experiment. ${ }^{5,10}$ We remind the reader that SEOS correlations, in the $L, M, N$, etc., shells cause shifts of a few $\mathrm{eV}^{4}$

(d) The symmetry adapted intershell pair energies of the hole (i.e., $1 s 2 s{ }^{3} S$, ${ }^{1} S$ ) increase significantly as we go from the ground to the excited state. Thus in spite of the fact that we have twice as many such interacting pairs of electrons in the ground state, the overall contribution of this intershell correlation energy to the BE is small. This fact holds for the hole-related intershell correlation energies in heavier atoms as well, but to a lesser degree.

Many-electron calculations. Within the framework of the theories of autoionizing states and atomic calculations presented elsewhere, ${ }^{1,15,16}$ we have carried out the following computations.

We have computed a correlated, square-integrable wave function $\Psi_{0}$, corresponding to the autoionizing state $\mathrm{Be}^{+} 1 s 2 s^{22} S$. First, we computed a multiconfigurational Hartree-Fock ${ }^{29}$ function for the Fermi sea configurations $1 s 2 s^{2}, 1 s 2 p^{2}$. The result is

$$
\begin{aligned}
& \Psi_{\mathrm{FS}}=0.94091 s 2 s^{2}+0.33871 s 2 p^{2}, \\
& E_{\mathrm{FS}}=\left\langle\Psi_{\mathrm{FS}}|H| \Psi_{\mathrm{FS}}\right\rangle=-10.10266,
\end{aligned}
$$

where $E_{\mathrm{FS}}$ is given in a.u. The Hartree-Fock energy for the single configuration $1 s 2 s^{2}$ is (in a.u.)

$$
E_{\mathrm{HF}}=\left\langle\Phi_{\mathrm{HF}}|H| \Phi_{\mathrm{HF}}\right\rangle=-10.04050 .
$$

The configuration $1 s^{2} 2 s$, which also belongs to the Fermi sea and corresponds to part of the $\sigma_{\mathrm{loc}}$ cluster of Eq. (3), is included during the computation of $\Psi_{0}$ and the corresponding energy of $E_{0}$. The configuration $1 s 2 p^{2}$ corresponds to part of $\pi_{\text {loc }}$ of Eq. (2).

Once $\Psi_{\mathrm{FS}}$ and $E_{\mathrm{FS}}$ have been computed, the remaining localized electron correlation is computed from a variational calculation, where the single-, pair-, or triple-orbital correlation functions satisfy, by symmetry or by Schmidt orthogonalization, the orthogonality constraint

$$
\left\langle\sigma, \text { or } \pi \text {, or } \tau / 1 s_{\mathrm{MCHF}}\right\rangle=0 \text {. }
$$

The necessity of the orthogonality constraint (6) in the $N$-electron theory of autoionizing states which uses a Hartree-Fock zeroth-order vector, and its conceptual as well as numerical limitations, have been introduced and discussed in Refs. 16 [see Eq. (44) of Ref. 16a] and 15.

The final square-integrable $\Psi_{0}$ contains only the localized correlation, which contributes to the stability of the state. In it is included the $1 s^{2} 2 s$ configuration which is part of the discrete Rydberg series and comes from the single orbital (de-) excitation $2 s \rightarrow 1 s$. Such single-orbital (de-) excitations into partly filled inner holes can contribute significantly in heavier systems, especially if they are of the $l \leftrightarrow l \pm 2$ type. $^{4}$

We note that the remainder of the Rydberg series is formed from pair (de-) excitations $2 s^{2} \rightarrow 1 s n s$. These can be included in the calculation of $\Psi_{0}$ and $E_{0}$, and their effect computed to all orders, ${ }^{16}$ or in the calculation of $\Delta .^{15}$ With the inclusion of $1 s^{2} 2 s$ in $\Psi_{0}$, their effect is negligible.

The number of configurations in $\Psi_{0}$ is 30 and the total energy (in a.u.)

$$
E_{0}=\left\langle\Psi_{0}|H| \Psi_{0}\right\rangle=-10.11980 .
$$


TABLE I. Breakdown of electron correlation of the $\mathrm{Be}^{+} 1 s 2 s^{22} S$ state in terms of one- and two-orbital localized and asymptotic correlations, after diagonalization. $v_{l}$ are virtual orbitals of $l$ symmetry. Zeroth-order $1 s, 2 s$, and $2 p$ orbitals are obtained from a MCHF calculation. Corresponding single-configuration $1 s 2 s^{2}$ energy is -10.04216 a.u. which is lower than the $\mathrm{HF}$ energy of Eq. (5) by $0.045 \mathrm{eV}$.

\begin{tabular}{|c|c|c|}
\hline Type of correlation & & Contribution (in $\mathrm{eV}$ ) \\
\hline $\begin{array}{l}\Phi_{\mathrm{HF}}^{-1} \sigma_{1 s}^{\text {loc }}, 1 s \rightarrow v_{s} \\
\Phi_{\mathrm{HF}}^{-1} \sigma_{2 s}^{\text {loc }}, 2 s \rightarrow 1 s, v_{s}\end{array}$ & & $\begin{array}{l}+0.0038 \\
-0.2084\end{array}$ \\
\hline$\Phi_{\mathrm{HF}}^{-2} \pi_{1 s 2 s}^{\mathrm{loc}}, 1 s 2 s \rightarrow v_{l} v_{l}^{\prime}, l=0,1,2,3$ & ${ }^{3} S$ & -0.0286 \\
\hline $\begin{array}{l}\Phi_{\mathrm{HF}}^{-2} \pi_{2 s^{2}}^{\mathrm{as}}, 2 s^{2} \rightarrow 2 p^{2}, 2 p v_{p}, v_{l} v_{l}^{\prime}, l=1,2,3 \\
\Phi_{\mathrm{HF}}^{-2} \pi_{2 s^{2}}^{\mathrm{as}}, 2 s^{2} \rightarrow 1 s \epsilon s\end{array}$ & ${ }^{1} S$ & $\begin{array}{l}-0.0661 \\
-1.8096 \\
-0.0408 \\
\end{array}$ \\
\hline Total & & -2.15 \\
\hline
\end{tabular}

We note that without the $1 s^{2} 2 s$ configuration, which is excluded from a projection-operator method such as that of Ref. 5, the total energy is $E_{0}^{\prime}=-10.122665$ a.u. compared with -10.12259 a.u. of Ref. 5. Comparing Eq. (7) with Eq. (5) we obtain for the localized correlation energy, $E_{\text {corr }}^{\text {loc }}=2.158 \mathrm{eV}$.

Having obtained $\Psi_{0}$ and $E_{0}$, we compute the energy shift $\Delta$ due to the asymptotic electron correlation of the $2 s^{2}$ electrons. The shift is given by the wellknown formula (e.g., Refs. 15 and 28) (the contribution of the Rydberg series is negligible beyond $1 s^{2} 2 s$ )

$$
\begin{aligned}
\Delta(E) & =P \int d \epsilon \frac{\left|\left\langle\Psi_{0}|H-E| U(\epsilon)\right\rangle\right|^{2}}{E-\epsilon} \\
& =-0.041
\end{aligned}
$$

given in $\mathrm{eV}$, where $P$ stands for principal value. In our case, $U(\epsilon)$ is the continuum Hartree-Fock function computed in the frozen $\mathrm{Be}^{2+} 1 s^{2}$ core. ${ }^{30}$ Equation (8) computes the contribution of $\pi_{\mathrm{as}}$ of Eq. (2) (there is no $\tau_{\text {as }}$ in the case of $\mathrm{Be}^{+}$) not only through the direct $\left\langle 2 s^{2}|H-E| 1 s \epsilon s\right\rangle$ interaction, but also via the higher-order indirect contributions of the localized correlation. Nonorthonormality is taken into account explicitly (see Sec. IV).

Adding Eqs. (8) and (7) we obtain the total nonrelativistic energy of the $\mathrm{Be}^{+} 1 s 2 s^{22} S$ state (in a.u.):

$$
E=E_{0}+\Delta=-10.12131 \text {. }
$$

Table I contains the breakdown of electron correlation in terms of the one- and two-orbital excited vectors which contribute to the energy $E_{0}$ and the shift $\Delta$, after diagonalization.

Given the calculation of $E$ and of the related symmetry-adapted pair energies, we can utilize published information for the ground state ${ }^{31-34}$ (see
Table II) to calculate the $1 \mathrm{~s} \mathrm{BE}$ of $\mathrm{Be}$ with two methods: (a) by taking the difference of the total energies and (b) by analyzing electron correlation for initial and final states in terms of the calculated symmetry-adapted pair energies and considering their individual effects on the BE.

(a) When the energy $E$ of Eq. (9) is combined with the nonrelativistic energy of the initial state (Table II), the resulting nonrelativistic $\mathrm{BE}$ is (in $\mathrm{eV}$ )

$$
\left(E_{B}\right)_{\mathrm{NR}}=123.705 \text {. }
$$

If to this number we add one-half the value (relativistic and radiative corrections) of Table II, i.e., $0.027 \mathrm{eV}$, we have the final theoretical prediction of this work (in $\mathrm{eV}$ ):

TABLE II. Published data for the Be ground state considered in this work ( 1 a. u. $=27.21165 \mathrm{eV}$ ) are the following. (a) Total nonrelativistic energy (Ref. 33) equals -14.667358 a.u., (b) relativistic and radiative energy (Ref. 33) equals -0.001987 a.u., (c) correlation energy (Ref. 33) equals $-2.56604 \pm 00068 \mathrm{eV}$, and (d) pair correlation energies ${ }^{a}$ (in a.u.).

\begin{tabular}{lccc}
\hline \hline & Reference 31 & Reference 32 & Reference 34 \\
\hline $1 s^{2}$ & -0.0425 & -0.0418 & -0.0421 \\
$1 s 2 s$ & -0.0052 & -0.0058 & -0.0041 \\
$2 s^{2}$ & -0.0448 & -0.0453 & -0.0461 \\
$1 s 2 p$ & & & -0.0004 \\
\hline \hline
\end{tabular}

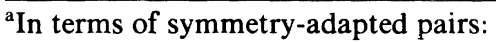

$$
\begin{aligned}
\epsilon(1 s 2 s) & =3 \times\left(1 s 2 s^{3} S\right)+1 s 2 s^{1} S \\
& =3 \times(0.00081)+(0.00342) \\
& =3 \times(0.00081)+(0.00281),
\end{aligned}
$$

where the latter two lines of the equation refer to Refs. 32 and 31 , respectively. 


$$
\left(E_{B}\right)_{\text {theor }}=123.732
$$

which can be compared with the experimental value of Bisgaard et al. ${ }^{12}$ (in eV),

$$
\left(E_{B}\right)_{\text {expt }}=123.6 \pm 0.1 \text {. }
$$

(b) Computing differences of accurate total energies is not always easy or even possible for arbitrary $N$-electron systems. An alternative approach, which corrects the predictions of the readily computable $\mathrm{HF}$ approximation, aims first at the systematic analysis of electron correlation and its influence on inner-electron spectroscopy and then at the $a b$ initio computation of those effects which are found necessary to compute. ${ }^{4,11}$ That this is possible is immediately derived from the successes of the HF theory in cases of unperturbed Rydberg transitions, or of highly ionized atoms, where there is nearly complete cancellation of electron correlation. In more complex systems, such analyses were first introduced by Sinanoglu and co-workers. ${ }^{24-27,35,36}$

The success of such an approach depends on the degree of satisfaction of the following two principles.

(1) For each state involved, find a suitable zerothorder approximation and the correlation corrections to it which are exclusively state dependent, and compute them accurately.

(2) Decompose most of the remaining orthogonal correlation which is state independent in terms of symmetry-adapted, bivirtual energies and compute them to all orders. The general methods for the construction and computation of these pair energies as well as for their symmetry-dependent coefficients were given in Ref. 37.

In the simple case of $\mathrm{Be}$, the bivirtual (allexternal $^{26}$ ) symmetry-adapted energies are $\epsilon\left(1 s^{2}\right), \epsilon\left(1 s 2 s{ }^{3} S,{ }^{1} S\right)$, and $\epsilon\left(2 s^{2}\right)$ and are obtained from pair functions which are orthogonal to the $1 s$, $2 s$, and $2 p$ Fermi sea orbitals.

For the ground state, we use the results of Table II. We assume (all values in $\mathrm{eV}$ )

$$
\begin{aligned}
& \epsilon\left(1 s^{2}\right)=1.150 ; \\
& \epsilon(1 s 2 s)=0.155 \text { and } 0.111,
\end{aligned}
$$

where HF $1 s$ and $2 s$, and MCHF $1 s$ and $2 s$ orbitals are used, respectively, and

$$
\epsilon^{*}\left(2 s^{2}\right)=0.035 \text { and } 0.065
$$

with HF and MCHF orbitals, respectively. $\epsilon^{*}\left(2 s^{2}\right)$ is obtained by subtracting from the total $\epsilon\left(2 s^{2}\right)$ the nontransferable $2 s^{2} \leftrightarrow 2 p^{2}$ pair correlation, ${ }^{26}$ given by a MCHF calculation as $1.189 \mathrm{eV}$.

For the $\mathrm{Be}^{+} 1 s 2 s^{22} S$ state we have obtained to all orders (in $\mathrm{eV}$ )

$$
\begin{aligned}
\epsilon(1 s 2 s) & =\frac{3}{2}\left({ }^{3} S\right)+\frac{1}{2}\left({ }^{1} S\right) \\
& =\frac{3}{2} 0.0235+\frac{1}{2} 0.181=0.126 \\
& =\frac{3}{2} 0.139+\frac{1}{2} 0.132=0.095
\end{aligned}
$$

with HF $1 s, 2 s, 2 p$ and MCHF $1 s, 2 s, 2 p$ orbitals for (14a) and (14b), respectively, and

$$
\begin{aligned}
\epsilon^{*}\left(2 s^{2}\right) & =0.208 \\
& =0.119,
\end{aligned}
$$

where HF and MCHF orbitals were used for (14c) and $(14 \mathrm{~d})$, respectively. Where, again, $\epsilon^{*}\left(2 s^{2}\right)$ does not include the near-degeneracy correlation, $2 s^{2} \leftrightarrow 2 p^{2}$, found to be $1.691 \mathrm{eV}$, or the continuum interaction with $1 s \epsilon S$ (see Table I).

The above results demonstrate the following.

(1) The near-degeneracy $2 s^{2} \leftrightarrow 2 p^{2}$ correlation increases significantly in going from $\mathrm{Be}$ to $\mathrm{Be}^{+} 1 s 2 s^{22} S$, due to a net increase in the effective nuclear charge. Compared with the HF BE, the corresponding decrease in the $\mathrm{BE}$ is $0.502 \mathrm{eV}$.

(2) The nonexistence of $\epsilon\left(1 s^{2}\right)$ in the upper state increases the $\mathrm{BE}$ by $1.150 \mathrm{eV}$.

(3) The single-orbital correlation in the excited state decreases the HF BE by $0.20 \mathrm{eV}$.

(4) The $1 s 2 s$ correlation is sensitive to the choice of the zeroth-order orbitals, both in $\mathrm{Be}$ and $\mathrm{Be}^{+} 1 s 2 s^{2}$. There is a difference in the results obtained using HF zeroth-order orbitals and MCHF zeroth-order orbitals. In either case, the $1 s 2 s$ correlation in the hole state is larger than one-half that of the ground state, due to the contraction of the $L$ shell in the presence of a larger effective nuclear charge. Using the MCHF result, we see that there is a net increase of the BE of only $0.016 \mathrm{eV}$.

(5) Finally, the increase of the effective $Z$ increases $\epsilon^{*}\left(2 s^{2}\right)$ in the hole state, with a resulting net decrease of the $\mathrm{BE}$ of $0.054 \mathrm{eV}$ (the results corresponding to the MCHF orbitals are used).

Thus, we see that even without the hole-filling, asymptotic pair correlation $2 s^{2} \leftrightarrow 1 s \in s$, there is, in general, a variety of correlation effects which compete when computing binding energies. Their relative importance depends on the system under consideration. For example, in the $\mathrm{Be}$ case the net result is a small increase of the SCF HF BE of 123.34 $\mathrm{eV}$ by $0.43 \mathrm{eV}$ to give $123.77 \mathrm{eV}$.

On the other hand, for the $K$-shell $\mathrm{BE}$ in $\mathrm{Ne}$, the increase from the DF BE due to electron correlation is $1.1 \mathrm{eV} .{ }^{11}$ Furthermore, the energy shift due to the orbital pair-Rydberg, continuum interaction has a wide range of positive or negative values-especially when SEOS-type interactions are allowed.

The results of our calculations on Be suggest that upon inner-shell ionization, the transferability of 
TABLE III. 1s $\mathrm{BE}$ of Be from various many-electron approaches and comparison with experiment (in $\mathrm{eV}$ ).

\begin{tabular}{|c|c|c|c|c|c|c|c|c|c|c|}
\hline $\begin{array}{l}\text { Experiment } \\
\text { (Ref. 12) }\end{array}$ & Koopman's & $\begin{array}{l}\text { This v } \\
\Delta \mathrm{SCF}\end{array}$ & $\begin{array}{l}\text { vork }^{\mathrm{a}} \\
\text { Eq. (15) }\end{array}$ & Eq. (11) & Ref. $5^{\text {b }}$ & Ref. $6^{c}$ & Ref. $7^{b}$ & Ref. $8^{b}$ & Ref. $9^{d}$ & Ref. $10^{\mathrm{b}}$ \\
\hline $123.6 \pm 0.1$ & 128.785 & 123.339 & 123.82 & 123.732 & $\begin{array}{c}123.671 \\
(123.698)\end{array}$ & $\begin{array}{l}123.866 \\
127.922\end{array}$ & 125.47 & 124.50 & 124.21 & $\begin{array}{c}123.661 \\
(123.668)\end{array}$ \\
\hline
\end{tabular}

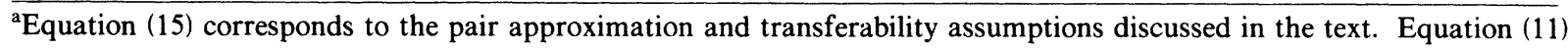
corresponds to total energy differences. Both include the relativistic and radiative corrections of $0.027 \mathrm{eV}$. Although the result of Eq. (11) is more accurate, Eq. (15) represents the result of a general and systematic approach applicable throughout the periodic table (Refs. 4 and 11 ).

bee text.

'See text. The second value corresponds to an extended Koopman's approach which used correlated ground-state wave functions.

${ }^{\mathrm{d}}$ See text. Obtained from the MCHF energy (-10.10266 a.u.), used in Ref. 9 to compute the position of Be ${ }^{+} 1 s 2 s^{22} S$ with respect to the $\mathrm{Be}^{+} 1 s^{2} 2 p, 3 p^{2} P^{o}$ states, and the Be ground-state energy of Table I.

certain pair correlations which are orthogonal to the state-dependent Fermi-sea and correlation vectors is not satisfied very well. This is caused by the increased attraction felt in the final state, especially when the hole is in the $K$ shell. On the other hand, when the requirement for accuracy is relaxed to, say, $\pm 0.3 \mathrm{eV}$, the cancellation of small errors allows successful predictions based on simpler calculations-as numerous applications on heavier systems have already demonstrated. ${ }^{4,11}$ For example, if we assumed that $\epsilon_{\mathrm{Be}}^{*}\left(2 s^{2}\right)=\epsilon_{\mathrm{Be}}^{*}+\left(2 s^{2}\right)$, used the MCHF $\epsilon\left(1 s^{2}\right)$ and $\epsilon\left(1 s 2 s^{3} S,{ }^{1} S\right)$ of Eq. (13), the near-degeneracy energies $2 s^{2} \leftrightarrow 2 p^{2}$ of $\mathrm{Be}(1.189 \mathrm{eV})$ and $\mathrm{Be}^{+}(1.691$ $\mathrm{eV}$ ), and the single-orbital excitation energies of Table I, we would obtain (in eV)

$$
\begin{aligned}
E_{B}= & \left(E_{\mathrm{MCHF}+\sigma+\Delta}\right)_{\text {final }} \\
& -\left(E_{\mathrm{MCHF}+\epsilon\left(1 s^{2}\right)+1 / 2 \epsilon(1 s 2 s)}\right)_{\text {initial }} \\
= & -275.15+398.95=123.80 .
\end{aligned}
$$

Our results for the binding energy are collected together in Table III, which also contains previous results of others (in Sec. V we compare the various results).

\section{THE AUGER WIDTH AND FLUORESCENCE OF $\mathrm{Be}^{+} 1 s 2 s^{22} S$}

Knowledge of $\Psi_{0}$ allows the calculation of the autoionization width and of the fluorescence yield from

$$
\Gamma\left(E_{0}\right)=2 \pi\left|\left\langle\Psi_{0}\left|H-E_{0}\right| U\left(E_{0}\right)\right\rangle\right|^{2}
$$

and

$$
\omega_{K}=\frac{\Gamma_{\mathrm{rad}}}{\Gamma_{\mathrm{rad}}+\Gamma_{\text {Auger }}},
$$

where $\Gamma_{\text {rad }}$ is the total radiative decay probability into all allowed lower states. Both $\Gamma_{\text {rad }}$ and $\Gamma_{\text {Auger }}$ are assumed to be energy independent so that they are given by the well-known "golden rule" expressions.

In calculating radiative or radiationless transition probabilities of $\mathrm{N}$-electron systems, existing theory and results $483,39,23$ indicate the following.

(a) The role of certain correlation effects is different as compared with total energy calculations.

(b) A better and more economical understanding of the physical or chemical process is obtained if the initial and final states are optimized separately. When this is done, the two basis sets are not orthonormal. Neglect of this nonorthonormality (NON) is often arbitrary, in which case there is an uncertainty in the accuracy of the computation.

As it has already been noted, ${ }^{20,23,37,39}$ our approach accounts for NON. In this work, the final continuum state $U\left(E_{0}\right)$ is represented by a frozen core HF scattering orbital in the field of $\mathrm{Be}^{2+} 1 s^{2}$. For the lower states of ${ }^{2} P^{o}$ symmetry we consider the $1 s^{2} 2 p$ and $1 s^{2} 3 p^{2} P^{o}$ states. Rough estimates showed that the decay rate to discrete $1 s^{2} n p, n>2$, and continuum $1 s^{2} \epsilon p$ states accounts for about $10 \%$ of the total.

Regarding the effects of electron correlation on the transition processes, we have the following comments.

(1) The configuration $1 s^{2} 2 s$ is important for the radiative decay rate. As it has already been pointed out in the analysis of autoionizing states, ${ }^{15,16}$ the hole-filling correlations which are left out in energy calculations using $P, Q$-type formalisms ${ }^{5,10}$ affect the wave function and related properties.

(2) Although the results of Kelly ${ }^{9}$ indicated that electron correlation increases the Auger width of $\mathrm{Be}^{+}$, this is not the case. The angular correlation of the $2 s^{2}$ electrons, as computed by the MCHF method, indeed increases the radiationless decay 
TABLE IV. Theoretical Auger widths for the $\mathrm{Be}^{+} 1 s 2 s^{2} S$ state (in eV), el. corr. stands for electron correlation.

\begin{tabular}{cccccc}
\hline \hline Reference $7^{\mathrm{a}}$ & Reference $10^{\mathrm{b}}$ & \multicolumn{2}{c}{ Reference $9^{\mathrm{c}}$} & \multicolumn{2}{c}{ This work $^{\mathrm{d}}$} \\
& & Hartree-Fock & MCHF [Eq. (3)] & MCHF [Eq. (3)] & MCHF plus el. corr. ( $\left.\Psi_{0}\right)$ \\
\hline 0.020 & 0.053 & 0.049 & 0.094 & 0.083 & 0.023 \\
\hline \hline
\end{tabular}

aGreen's function method with Siegert boundary conditions.

${ }^{b}$ Projected configuration interaction for the initial state. Golden rule formula.

The MCHF function of Eq. (3) and the golden rule formula. Nonorthonormality has been neglected.

Nonorthonormality between initial- and final-state vectors is computed explicitly, for MCHF as well as for $\Psi_{0}$. See text.

rate. However, radial correlation $2 s^{2} \leftrightarrow v_{s}^{2}$, and spin polarization $1 s 2 s^{2} \leftrightarrow 1 s 2 s v_{s}{ }^{3} S$, contribute with an opposite sign and reduce the width. The contribution of these major effects to the matrix element of Eq. (16) is $1 s 2 s^{2}=-0.01886$ a.u., $1 s 2 p^{2}=-0.00317$ a.u., $1 s v_{s}^{2}=0.00242$ a.u., and $1 s 2 s v_{s}^{3} S=0.00823$ a.u.

Here we note that, in an analysis of the effect of electron correlation on two-electron autoionizing states, Moiseyev and Weinhold ${ }^{40}$ concluded that the angular correlation should decrease the width. In the case of $\mathrm{Be}^{+}$, Kelly's and our results show the opposite trend.

(3) Both at the HF and full correlation level, NON plays a role. This is demonstrated by comparing our and Kelly's results for the HF and MCHF radiative and radiationless transitions. They are different. Especially for the radiative decay, the difference has even conceptual implications, since we show that there is a nonzero transition probability even at the HF level and thus radiative decay does not arise only from correlation effects. In fact, the radial matrix element of the $1 s 2 s^{2} \rightarrow 1 s^{2} 2 p$ transition is 0.0245 a.u. compared to 0.0642 a.u. for that of the $1 s 2 p^{2} \rightarrow 1 s^{2} 2 p$ transition, using the MCHF function of Eq. (3).

For the autoionization width, NON is caused primarily by the overlap of the scattering $\epsilon s$ orbital of the final state with certain bound orbitals of the initial state and has the effect of introducing some otherwise forbidden one-body and $R^{k}$ integrals. For the $1 s 2 s^{2}$ configuration, in addition to the two-electron interaction, a significant term containing the onebody integral $-I(1 s, 2 s)$ times the overlap $S(2 s, \epsilon s)$ appears.

The results of our calculations are presented in Tables IV and V together with those of other theories and experiment. The experimental $\omega_{K}$ is measured in the solid state.

\section{COMPARISON WITH OTHER THEORETICAL RESULTS}

The Be $1 s$ BE, Auger width, and fluorescence yield have been studied before via different manyelectron theoretical approaches. ${ }^{5-10}$ The results of these calculations are collected in Tables III-V together with those of the present work.

In order to present the BE's for Refs. 5, 6, and 10, we have used their published total nonrelativistic en-

TABLE V. Radiative decay rates (in $\sec ^{-1}$ ) of $\mathrm{Be}^{+} 1 s 2 s^{2} S$ and fluorescence yield $\left(\omega_{K}\right)$.

\begin{tabular}{|c|c|c|c|c|c|}
\hline Transition & Reference 9 & $\mathrm{HF}^{\mathrm{a}}$ & $\begin{array}{r}\text { This } \\
\mathrm{MCHF}^{\mathrm{a}}\end{array}$ & $\begin{array}{l}\text { work } \\
\text { MCHF plus corr. }\end{array}$ & Expt. $^{b}$ \\
\hline $1 s^{2} 2 p$ & $3.03 \times 10^{9}$ & $5.3 \times 10^{9}$ & $17.5 \times 10^{9}$ & $3.73 \times 10^{9}$ & \\
\hline $1 s^{2} 3 p$ & $0.13 \times 10^{9}$ & & & $0.22 \times 10^{9}$ & \\
\hline$\omega_{K}$ & $0.2238 \times 10^{-4}$ & & & $1.2 \times 10^{-4 c}$ & $\begin{array}{l}3.04 \times 10^{-4} \pm 20 \% \text { (Ref. 13) } \\
3.6 \times 10^{-4} \pm 30 \% \quad \text { (Ref. 14) }\end{array}$ \\
\hline
\end{tabular}

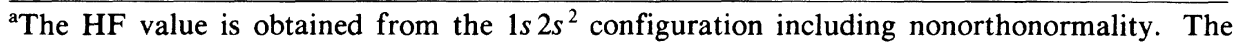
finite value implies that radiative decay is not due only to electron correlation (Ref. 9). Thus, it is demonstrated again that multielectron transitions can be described semiquantitatively from the true HF transition theory (Refs. 23 and 39). For HF and MCHF, the length form is adopted. For the fully correlated result, all three forms agree (Refs. 39 and 41).

${ }^{b}$ Refers to the solid state. A discussion of the causes of observed deviation between the atomic and solid state is given in Ref. 9.

${ }^{\mathrm{c}}$ To the calculated transition probability from the $1 s 2 s^{22} S \rightarrow 1 s^{2} 2 p^{2} P^{o}$ transition we have added an estimated $10 \%$ contribution to $\omega_{K}$ from the Rydberg series $1 s^{2} n p, n>2$, and continuum $1 s^{2} \epsilon p$. 
ergy of the $\mathrm{Be}^{+}$state and subtracted from it the ground-state energy of Table I. For reasons of comparison with experiment and our work, we also give a second number (in parentheses) for Refs. 5 and 10, which is obtained by adding the relativistic correction of $0.027 \mathrm{eV}$.

In Ref. 6 two types of calculations are reported. One is a straightforward total configurationinteraction calculation which yields an energy for the hole state of -10.11541 a.u. The other is called the "extended Koopman's" method and yields the BE with the use of only correlated ground-state functions. The data of Table III suggest that the extended Koopman's method-at least for $\mathrm{Be}$-is closer to the actual Koopman's theorem results than to experiment. Apparently relaxation is not well accounted for.

Reference 8 presents the BE result from an application of a Green's-function technique. The deviation from the experimental value is attributed by the authors to basis set limitations.

The results of Ref. 7 are obtained from a Green's-function technique using a basis set with Siegert boundary conditions. This calculation yielded a BE which differs substantially from those of our calculations and those obtained with other methods. This is expected since the approximations employed in Ref. 7 do not account for relaxation and much of electron correlation. On the other hand, the width is close to ours. This is fortuitous.

Kelly ${ }^{9}$ has employed the MCHF energy of Eq. (4) for the calculation of the transition energies to the $\mathrm{Be}^{+} 1 s^{2} 2 p, 3 p$ states, whose total energy was calculated by adding to the HF energy the $1 s^{2}$ correlation energy. The MCHF wave function of Eq. (3) was employed for the calculation of the Auger width and many-body perturbation theory was employed for the calculation of the radiative transition probabilities.

The effect of the remaining electron correlation in the excited state on the observables was not studied. Kelly's calculations did not account for NON and this is why his HF values differ from ours.

The results of Bhatia ${ }^{10}$ and Davis and Chung ${ }^{5}$ are obtained from large configuration-interaction calculations on projected spaces. Their variational methods, in which specific orbital functions are projected out of the trial $N$-electron function, are similar to the one employed in this work. We point out that the theoretical foundations of such variational calculations on arbitrary $N$-electron autoionizing states are present in Ref. 16. For example, Eqs. (1) and (5) of Ref. 5, where ${ }^{41}$ "saddle-point technique" is applied, follow from Eqs. (35), (44), and (45) of Ref. 16. Here we note that the orthogonality con- straints to fixed" 16 or "optimized"5 orbital functions are not rigourous to all orders, though they are sufficiently accurate. For, as we demonstrated in Ref. 15 , even for two-electron systems the rigorous and unique definition of projection operators in practical applications of Rayleigh-Ritz-type variational calculus is very difficult.

We close by noting that the total energies of Refs. 5 and 10 do not include the energy shift due to the perturbation of the continuum [our Eq. (8)] or of the Rydberg series which belong to the discrete spectrum (i.e., $1 s^{2} n s^{2} S$ ), nor a correction for relativistic and radiative corrections. When we add to their results $0.027 \mathrm{eV}$ for the latter quantities, the BE's become $123.698 \mathrm{eV}$ (Ref. 5) and 123.668 eV (Ref. 10).

\section{CONCLUSION}

The results of this work suggest the following.

(1) The $K$-shell BE, Auger width, and fluorescence yield of Be in the $1 s 2 s^{22} S$ state are $123.73 \mathrm{eV}$, $0.023 \mathrm{eV}$, and $1.2 \times 10^{-4}$, respectively. Our $\omega_{K}$ value implies that the atomic character of fluorescence in the solid is much larger than previously predicted. ${ }^{9}$

(2) The analysis of bound and autoionizing states in terms of Hartree-Fock and symmetry-adapted one- and two-subshell localized and asymptotic correlation allows the systematic and accurate interpretation of core-electron spectra. The Be case is relatively simple and is tractable in terms of total energies and wave functions. However, this does not apply to larger systems, where the identification and consistent calculation of specific correlation effects are prerequisites for quantitative work. (Reference 4 and references therein.)

(3) The continuum effects in $\mathrm{Be}^{+} 1 s 2 s^{22} S$ are weak. The corresponding energy shift is -0.041 $\mathrm{eV}$. In calculating $\Delta$, only the scattering states $1 s^{2} \epsilon S$ were considered. The contribution of the Rydberg series $1 s^{2} n s$ was included to all orders in the calculation of $\Psi_{0}$, via the inclusion of the $1 s^{2} 2 s$ configuration which comes from the single-subshell (de-) excitation $2 s \rightarrow 1 s$.

Since it lies below the energy of the autoionizing state, the variational upper bound procedure remains valid. We note that, provided that near degeneracy of roots is avoided, hole-filling pair correlations which give rise to the Rydberg and continuum series can be represented with reasonable success with square-integrable functions [e.g., see Ref. 16(a), p. 2088, Ref. 16(b), p. 237, and Refs. 15, 4, and 42].

(4) The creation of a hole in the $K$ shell affects the state specificity of symmetry-adapted $K-L$ and $L$ shell pair correlations. This effective charge effect 
holds throughout the periodic table but to different degrees, depending on the position of the hole and the electronic structure of the system in question.

(5) Fermi-sea correlations of the valence shell are not sufficient to produce accurate widths. In fact, here, the angular correlation $2 s^{2} \leftrightarrow 2 p^{2}$ worsens the HF result. When additional, radial and spinpolarization correlations are considered, the width is reduced considerably

(6) Nonorthonormality is shown again ${ }^{20,39}$ to be quantitatively important in the theory of radiative and radiationless transitions. With NON present, transitions which have been though of as forbidden at the independent-particle level, can be appreciably nonzero. ${ }^{23,42}$
${ }^{1}$ Photoemission in Solids, edited by L. Ley and M. Cardona (Springer, Berlin, 1978), Vols. I and II.

${ }^{2}$ M. O. Krause, in Synchrotron Radiation Research, edited by $\mathbf{H}$. Winick and S. Doniach (Plenum, New York 1980), p. 101.

${ }^{3} X$-Ray Photoelectron Spectroscopy, edited by T. A. Carlson (Dowden, Hutchinson and Ross, Stroudsburg, 1978).

${ }^{4}$ D. R. Beck and C. A. Nicolaides, Phys. Rev. A $\underline{26}, 857$ (1982).

${ }^{5}$ B. F. Davis and K. T. Chung, J. Phys. B 15 , 3113 (1982).

${ }^{6}$ R. C. Morrison, Chem. Phys. Lett. 62, 131 (1979).

${ }^{7}$ M. Palmquist, P. L. Altick, J. Richter, P. Winkler, and R. Yaris, Phys. Rev. A 23 1795 (1981).

${ }^{8}$ O. Walter and J. Schirmer, J. Phys. B 14, 3805 (1981).

${ }^{9}$ H. P. Kelly, Phys. Rev. A 9, 1582 (1974).

${ }^{10}$ A. K. Bhatia, Phys. Rev. A $\underline{18}, 2523$ (1978).

${ }^{11}$ D. R. Beck and C. A. Nicolaides, in Excited States in Quantum Chemistry, edited by C. A. Nicolaides and D. R. Beck (Reidel, Dordecht, 1979), p. 329.

12P. Bisgaard, R. Bruch, P. Dahl, B. Fastrup, and M. Rødbro, Phys. Scr. 17, 49 (1978).

${ }^{13}$ C. E. Dick and A. C. Lucas, Phys. Rev. A 2 580 (1970).

${ }^{14}$ K. Feser, Phys. Rev. Lett. 28, 1013 (1972).

${ }^{15}$ C. A. Nicolaides and D. R. Beck, Int. J. Quantum Chem. 14, 457 (1978).

${ }^{16}$ C. A. Nicolaides, Phys. Rev. A $\underline{6}, 2078$ (1972); Nucl. Instrum. Methods 110, 231 (1973).

${ }^{17}$ C. A. Nicolaides and D. R. Beck, Phys. Rev. A $\underline{18}, 1307$ (1978).

${ }^{18}$ See, e.g., W. H. E. Schwarz and H. Wallmeier, Mol. Phys. 46, 20 (1982).

${ }^{19}$ C. A. Nicolaides, Y. Komninos, and Th. Mercouris, Int. J. Quantum Chem. Symp. 15, 355 (1981).

${ }^{20}$ C. A. Nicolaides, Y. Komninos, and D. R. Beck, Phys. Rev. A 24, 1103 (1981).

${ }^{21}$ D. R. Beck and C. A. Nicolaides, Chem. Phys. Lett. $\underline{59}$, 525 (1978).
${ }^{22}$ D. R. Beck, Int. J. Quantum Chem. Symp. 16, 345 (1982).

${ }^{23}$ Y. Komninos, G. Aspromallis, and C. A. Nicolaides, Phys. Rev. A 27, 1865 (1983).

${ }^{24}$ O. Sinanoglu, Adv. Chem. Phys. $\underline{6}$, 315 (1964).

${ }^{25}$ V. McKoy and O. Sinanoglu, J. Chem. Phys. 41, 2689 (1964).

26I. Öksuz and O. Sinanoglu, Phys. Rev. 181, 42 (1969).

${ }^{27}$ C. A. Nicolaides, Chem. Phys. Lett. 19, 67 (1973); C. A. Nicolaides and D. R. Beck, Chem. Phys. Lett. 27, 269 (1974).

${ }^{28}$ U. Fano, Phys. Rev. 124, 1866 (1961).

${ }^{29}$ C. Froese-Fischer, Comput. Phys. Commun. 4, 107 (1972).

${ }^{30}$ G. N. Bates, Comput. Phys. Commun. $\underline{8}, 220$ (1974).

${ }^{31}$ F. W. Byron and C. J. Joachain, Phys. Rev. 157, 7 (1967).

${ }^{32}$ R. K. Nesbet, Phys. Rev. 155, 51 (1967).

${ }^{33}$ C. F. Bunge, Phys. Rev. A 14, 1965 (1976).

${ }^{34}$ C. Froese-Fischer and K. M. S. Saxena, Phys. Rev. A 9, 1498 (1974).

${ }^{35}$ H. J. Silverstone and O. Sinanoglu, J. Chem. Phys. 44, 1898 (1966).

${ }^{36}$ C. A. Nicolaides and D. R. Beck, J. Phys. B 6,535 (1973).

${ }^{37}$ D. R. Beck and C. A. Nicolaides, in Excited States in Quantum Chemistry, edited by C. A. Nicolaides and D. R. Beck (Reidel, Dordrecht, 1979), p. 105.

${ }^{38}$ C. A. Nicolaides and D. R. Beck, Chem. Phys. Lett. $\underline{36}$, 79 (1975).

${ }^{39}$ C. A. Nicolaides and D. R. Beck, in Excited States in Quantum Chemistry, edited by C. A. Nicolaides and D. R. Beck (Reidel, Dordrecht, 1979), p. 143.

${ }^{40}$ N. Moiseyev and F. Weinhold, Phys. Rev. A 20, 27 (1979).

${ }^{41}$ K. T. Chung, Phys. Rev. A $\underline{23}, 1079$ (1981).

${ }^{42}$ C. A. Nicolaides and D. R. Beck, J. Chem. Phys. 66, 1982 (1977). 